\title{
ON THE FOURIER TRANSFORM AND THE EXCHANGE PROPERTY
}

\author{
DRAGU ATANASIU AND PIOTR MIKUSIŃSKI
}

Received 19 June 2005

A simplified construction of tempered Boehmians is presented. The new construction shows that considering delta sequences and convergence arguments is not essential.

\section{Introduction}

Since Boehmians were introduced, extensions of the Fourier transform to spaces of Boehmians attracted a lot of attention (see [2, 3, 4, 5, 6, 7, 8, 9]). In some cases, the range of the extended Fourier transform is a space of functions. In other constructions, the range is a space of distributions or a space of Boehmians.

In this paper, we would like to consider the space of tempered Boehmians presented in [8]. In this case, the range of the Fourier transform is the space of all distributions $\mathscr{D}^{\prime}$. This work is motivated by [1].

First we recall briefly the construction of the space of tempered Boehmians. A continuous function $f: \mathbb{R}^{N} \rightarrow \mathbb{C}$ is called slowly increasing if there is a polynomial $p$ on $\mathbb{R}^{N}$ such that $|f(x)| \leq p(x)$ for all $x \in \mathbb{R}^{N}$. The space of slowly increasing functions will be denoted by $\mathcal{W}\left(\mathbb{R}^{N}\right)$ or simply $\mathcal{W}$.

An infinitely differentiable function $f: \mathbb{R}^{N} \rightarrow \mathbb{C}$ is called rapidly decreasing if

$$
\sup _{|\alpha| \leq m} \sup _{x \in \mathbb{R}^{N}}\left(1+x_{1}^{2}+\cdots+x_{N}^{2}\right)^{m}\left|D^{\alpha} f(x)\right|<\infty
$$

for every nonnegative integer $m$, where $x=\left(x_{1}, \ldots, x_{N}\right), \alpha=\left(\alpha_{1}, \ldots, \alpha_{N}\right), \alpha_{n}$ 's are nonnegative integers, $|\alpha|=\alpha_{1}+\cdots+\alpha_{N}$, and

$$
D^{\alpha}=\frac{\partial^{|\alpha|}}{\partial x^{\alpha}}=\frac{\partial^{|\alpha|}}{\partial x_{1}^{\alpha_{1}} \cdots \partial x_{N}^{\alpha_{N}}} .
$$

The space of rapidly decreasing functions is denoted by $\mathscr{Y}\left(\mathbb{R}^{N}\right)$ or simply $\mathscr{I}$.

If $f \in \mathscr{W}$ and $\varphi \in \mathscr{Y}$, then the convolution

$$
f * \varphi(x)=\int_{\mathbb{R}^{N}} f(y) \varphi(x-y) d y
$$

is well defined and $f * \varphi \in \mathcal{W}$. 
A sequence $\left(\varphi_{n}\right) \in \mathscr{G}^{\mathbb{N}}$ is called a delta sequence if it satisfies the following conditions:

(a) $\int_{\mathbb{R}^{N}} \varphi_{n}(x) d x=1$ for all $n \in \mathbb{N}$,

(b) $\int_{\mathbb{R}^{N}}\left|\varphi_{n}(x)\right| d x \leq C$ for some constant $C$ and all $n \in \mathbb{N}$,

(c) $\lim _{n \rightarrow \infty} \int_{\|x\| \geq \varepsilon}\|x\|^{k}\left|\varphi_{n}(x)\right| d x=0$ for every $k \in \mathbb{N}$ and $\varepsilon>0$.

The space of tempered Boehmians $\mathscr{B}$ is defined as the space of equivalence classes of pairs of sequences $\left(f_{n}, \varphi_{n}\right)$, where $f_{n} \in \mathcal{W}$ and $\left(\varphi_{n}\right)$ is a delta sequence as defined above, satisfying

$$
f_{m} * \varphi_{n}=f_{n} * \varphi_{m} \quad \forall m, n \in \mathbb{N}
$$

with respect to the equivalence relation defined by

$$
\left(f_{n}, \varphi_{n}\right) \sim\left(g_{n}, \gamma_{n}\right) \quad \text { if } f_{m} * \gamma_{n}=g_{n} * \varphi_{m}, \forall m, n \in \mathbb{N} .
$$

It is shown in [8] that the Fourier transform can be defined for tempered Boehmians and that the range is exactly the space of all distributions $\mathscr{D}^{\prime}$. Thus, the space of tempered Boehmians can be identified with the space of ultradistributions $\mathscr{L}^{\prime}$ (see, e.g., [10]). In the construction, the particular choice of delta sequences and the fact that the Fourier transform of a delta sequence converges to 1 uniformly on compact subsets of $\mathbb{R}^{N}$ seem to be essential. In this paper, we show that this is not the case. In fact, we give an equivalent construction where convergence plays no role. This approach indicates that the results of [8] follow from a more general principle.

In what follows, we will denote by $\mathscr{S}^{\prime}$ the space of tempered distributions, that is, the space of continuous linear functionals on $\mathscr{S}$. If $f \in \mathscr{S}^{\prime}$ and $\varphi \in \mathscr{Y}$, then the convolution $f * \varphi$ is defined as $(f * \varphi)(x)=f\left(\varphi_{x}\right)$, where $\varphi_{x}(z)=\varphi(x-z)$. It can be shown that, if $f \in \mathscr{S}^{\prime}$ and $\varphi \in \mathscr{Y}$, then $f * \varphi \in \mathcal{W}$. The Fourier transform of a tempered distribution $f$, denoted by $\hat{f}$, is the functional on $\mathscr{S}$ defined by $\hat{f}(\varphi)=f(\hat{\varphi})$, where $\hat{\varphi}$ is the Fourier transform of $\varphi$.

\section{The exchange property}

For a family $\left\{\varphi_{j}\right\}_{j \in J}=\left\{\varphi_{j}\right\}_{J}$, where $J$ is an index set and $\varphi_{j} \in \mathscr{Y}$ for all $j \in J$, we define

$$
M\left(\left\{\varphi_{j}\right\}_{J}\right)=\left\{x \in \mathbb{R}^{N}: \hat{\varphi}_{j}(x)=0 \forall j \in J\right\} .
$$

A family of pairs $\left\{\left(f_{j}, \varphi_{j}\right)\right\}_{J}$, where $f_{j} \in \mathscr{S}^{\prime}$ and $\varphi_{j} \in \mathscr{Y}$, is said to have the exchange property if

$$
f_{j} * \varphi_{k}=f_{k} * \varphi_{j} \quad \forall j, k \in J .
$$

Theorem 2.1. If $\left\{\left(f_{j}, \varphi_{j}\right)\right\}_{J}$ has the exchange property and $\Omega=M\left(\left\{\varphi_{j}\right\}_{J}\right)^{c}$ (the complement of $M\left(\left\{\varphi_{j}\right\}_{J}\right)$ in $\left.\mathbb{R}^{N}\right)$, then there exists a unique $F \in \mathscr{D}^{\prime}(\Omega)$ such that

$$
\hat{f}_{j}=F \hat{\varphi}_{j} \quad \forall j \in J .
$$

Proof. For every $x \in \Omega$ there exists a $j \in J$ and $\varepsilon>0$ such that $\left|\hat{\varphi}_{j}(x)\right|>\varepsilon$ in an open neighborhood of $x$. Then we can define $F=\hat{f}_{j} / \hat{\varphi}_{j}$ in that neighborhood. We need to show 
that this definition of $F$ is independent of $j$. Suppose, for some $\varepsilon>0$, we have $\left|\hat{\varphi}_{j}(x)\right|>\varepsilon$ for all $x \in U$ and $\left|\hat{\varphi}_{k}(x)\right|>\varepsilon$ for all $x \in V$. Then, since $f_{j} * \varphi_{k}=f_{k} * \varphi_{j}$, we have $\hat{f}_{j} \hat{\varphi}_{k}=$ $\hat{f}_{k} \hat{\varphi}_{j}$ and

$$
\frac{\hat{f}_{j}}{\hat{\varphi}_{j}}=\frac{\hat{f}_{k}}{\hat{\varphi}_{k}}
$$

on $U \cap V$. Clearly, $F$ is unique.

We will denote by $\mathscr{A}$ the collection of all families of pairs $\left\{\left(f_{j}, \varphi_{j}\right)\right\}_{J}$, where $J$ is an index set, $f_{j} \in \mathscr{S}^{\prime}$ and $\varphi_{j} \in \mathscr{S}$ for all $j \in J$, satisfying the exchange property and such that $M\left(\left\{\varphi_{j}\right\}_{J}\right)=\varnothing$.

Note that in the definition of $\mathscr{A}$ the index set is not fixed. If $f \in \mathscr{Y}^{\prime}$ is arbitrary and $\omega(x)=e^{-x \cdot x}$, then $\{(f, \omega)\} \in \mathscr{A}$. In this case the index set has only one element.

If $\left(\varphi_{j}\right)$ is a delta sequence, then obviously $M\left(\left\{\varphi_{j}\right\}_{\mathbb{N}}\right)=\varnothing$. However, it is possible that $M\left(\left\{\varphi_{j}\right\}_{J}\right)=\varnothing$ and $\left\{\varphi_{j}\right\}$ does not contain any subsequence which is a delta sequence. Consider, for example, a sequence $\left\{\varphi_{j}\right\}_{\mathbb{N}}$ such that $\left\{\hat{\varphi}_{j}\right\}_{\mathbb{N}}$ is a partition of unity. More generally, let $\left\{U_{j}\right\}_{J}$ be an open covering of $\mathbb{R}^{N}$ and let $\left\{\varphi_{j}\right\}_{J}$ be such that $\left|\hat{\varphi}_{j}(x)\right|>0$ for $x \in U_{j}$. A family $\left\{\varphi_{j}\right\}_{J}$ such that $M\left(\left\{\varphi_{j}\right\}_{J}\right)=\varnothing$ will be called total.

Lemma 2.2. If $\left\{\varphi_{j}\right\}_{J}$ and $\left\{\gamma_{k}\right\}_{K}$ are total, then $\left\{\varphi_{j} * \gamma_{k}\right\}_{J \times K}$ is total.

Theorem 2.3. $\left\{\left(f_{j}, \varphi_{j}\right)\right\}_{J} \in \mathscr{A}$ if and only if there exists a unique $F \in \mathscr{D}^{\prime}\left(\mathbb{R}^{N}\right)$ such that $\hat{f}_{j}=\hat{\varphi}_{j} F$ for all $j \in J$.

Proof. We only need to show that existence of such an $F \in \mathscr{D}^{\prime}\left(\mathbb{R}^{N}\right)$ implies the exchange property. Indeed, for any $j, k \in J$ we have

$$
\hat{f}_{j} \hat{\varphi}_{k}=F \hat{\varphi}_{j} \hat{\varphi}_{k}=F \hat{\varphi}_{k} \hat{\varphi}_{j}=\hat{f}_{k} \hat{\varphi}_{j}
$$

Definition 2.4. If $\left\{\left(f_{j}, \varphi_{j}\right)\right\}_{J} \in \mathscr{A}$, then the unique $F \in \mathscr{D}^{\prime}\left(\mathbb{R}^{N}\right)$ such that $\hat{f}_{j}=\hat{\varphi}_{j} F$ for all $j \in J$ will be denoted by $\mathscr{F}\left(\left\{\left(f_{j}, \varphi_{j}\right)\right\}_{J}\right)$ and called the Fourier transform of $\left\{\left(f_{j}, \varphi_{j}\right)\right\}_{J}$.

Theorem 2.5. For every $F \in \mathscr{D}^{\prime}\left(\mathbb{R}^{N}\right)$ there exists $\left\{\left(f_{j}, \varphi_{j}\right)\right\}_{J} \in \mathscr{A}$ such that $F=\mathscr{F}\left(\left\{\left(f_{j}\right.\right.\right.$, $\left.\left.\left.\varphi_{j}\right)\right\}_{J}\right)$.

Proof. Let $\left\{\varphi_{j}\right\}_{\mathbb{N}}$ be a total sequence such that $\hat{\varphi}_{j} \in \mathscr{D}\left(\mathbb{R}^{N}\right)$ for all $j \in \mathbb{N}$, where $\mathscr{D}\left(\mathbb{R}^{N}\right)$ denotes the space of smooth functions with compact support. Then, for every $j \in \mathbb{N}$, there is an $f_{j} \in \mathscr{G}^{\prime}$ such that $\hat{f}_{j}=\hat{\varphi}_{j} F$. Clearly, $\left\{\left(f_{j}, \varphi_{j}\right)\right\}_{\mathbb{N}} \in \mathscr{A}$ and $F=\mathscr{F}_{(}\left(\left\{\left(f_{j}, \varphi_{j}\right)\right\}_{\mathbb{N}}\right)$.

Let $\left\{\left(f_{j}, \varphi_{j}\right)\right\}_{J},\left\{\left(g_{k}, \gamma_{k}\right)\right\}_{K} \in \mathscr{A}$. If $f_{j} * \gamma_{k}=g_{k} * \varphi_{j}$ for all $j \in J$ and $k \in K$, then we write $\left\{\left(f_{j}, \varphi_{j}\right)\right\}_{J} \sim\left\{\left(g_{k}, \gamma_{k}\right)\right\}_{K}$. This relation is clearly symmetric and reflexive. We will show that it is also transitive.

Let $\left\{\left(f_{j}, \varphi_{j}\right)\right\}_{J},\left\{\left(g_{k}, \gamma_{k}\right)\right\}_{K},\left\{\left(h_{l}, \psi_{l}\right)\right\}_{L} \in \mathscr{A}$. If $\left\{\left(f_{j}, \varphi_{j}\right)\right\}_{J} \sim\left\{\left(g_{k}, \gamma_{k}\right)\right\}_{K}$ and $\left\{\left(g_{k}, \gamma_{k}\right)\right\}_{K} \sim$ $\left\{\left(h_{l}, \psi_{l}\right)\right\}_{L}$, then

$$
f_{j} * \gamma_{k}=g_{k} * \varphi_{j}, \quad g_{k} * \psi_{l}=h_{l} * \gamma_{k}
$$


2582 On the Fourier transform and the exchange property

for all $j \in J, k \in K, l \in L$. Hence

$$
f_{j} * \gamma_{k} * \psi_{l}=g_{k} * \varphi_{j} * \psi_{l}, \quad g_{k} * \psi_{l} * \varphi_{j}=h_{l} * \gamma_{k} * \varphi_{j}
$$

for all $j \in J, k \in K, l \in L$. Since $*$ is commutative, we have

$$
f_{j} * \psi_{l} * \gamma_{k}=h_{l} * \varphi_{j} * \gamma_{k}
$$

for all $j \in J, k \in K, l \in L$. Now fix $j \in J$ and $l \in L$. Since $M\left(\left\{\gamma_{k}\right\}_{K}\right)=\varnothing$ and (2.8) holds for every $k \in K$, we conclude that $f_{j} * \psi_{l}=h_{l} * \varphi_{j}$ for all $j \in J$ and $l \in L$, which means that $\left\{\left(f_{j}, \varphi_{j}\right)\right\}_{J} \sim\left\{\left(h_{l}, \psi_{l}\right)\right\}_{L}$.

Note that

$$
\left\{\left(f_{j}, \varphi_{j}\right)\right\}_{J} \sim\left\{\left(f_{j} * \psi_{k}, \varphi_{j} * \psi_{k}\right)\right\}_{J \times K}
$$

for any total family $\left\{\psi_{k}\right\}_{K}$.

Theorem 2.6. Let $\left\{\left(f_{j}, \varphi_{j}\right)\right\}_{J},\left\{\left(g_{k}, \gamma_{k}\right)\right\}_{K} \in \mathcal{A}$. Then

$$
\left\{\left(f_{j}, \varphi_{j}\right)\right\}_{J} \sim\left\{\left(g_{k}, \gamma_{k}\right)\right\}_{K} \quad \text { iff } \mathscr{F}\left(\left\{\left(f_{j}, \varphi_{j}\right)\right\}_{J}\right)=\mathscr{F}\left(\left\{\left(g_{k}, \gamma_{k}\right)\right\}_{K}\right) .
$$

Proof. Let $F=\mathscr{F}_{(}\left(\left\{\left(f_{j}, \varphi_{j}\right)\right\}_{J}\right)$ and $G=\mathscr{F}\left(\left\{\left(g_{k}, \gamma_{k}\right)\right\}_{K}\right)$.

If $\left\{\left(f_{j}, \varphi_{j}\right)\right\}_{J} \sim\left\{\left(g_{k}, \gamma_{k}\right)\right\}_{K}$, then

$$
F \widehat{\varphi}_{j} \hat{\gamma}_{k}=\hat{f}_{j} \hat{\gamma}_{k}=\hat{g}_{k} \hat{\varphi}_{j}=G \hat{\gamma}_{k} \hat{\varphi}_{j}
$$

for all $j \in J$ and $k \in K$. Hence $F=G$, by Lemma 2.2.

Now assume $F=G$. Then

$$
\hat{f}_{j} \hat{\gamma}_{k}=F \hat{\varphi}_{j} \hat{\gamma}_{k}=G \hat{\gamma}_{k} \hat{\varphi}_{j}=\hat{g}_{k} \hat{\varphi}_{j}
$$

for all $j \in J$ and $k \in K$. Hence $\left\{\left(f_{j}, \varphi_{j}\right)\right\}_{J} \sim\left\{\left(g_{k}, \gamma_{k}\right)\right\}_{K}$.

Now we define $\mathscr{B}=\mathscr{A} / \sim$, the space of equivalence classes. In view of Theorems 2.5 and 2.6, the Fourier transform is a bijection from $\mathscr{B}$ to $\mathscr{D}^{\prime}$. Consequently, $\mathscr{B}$ can be identified with the space of ultradistributions $\mathscr{E}^{\prime}$. We will show that, with a properly defined convergence in $\mathscr{B}$, the spaces are isomorphic.

Note that the space $\mathscr{S}^{\prime}$ can be identified with a subspace of $\mathscr{B}$ via $f \mapsto[\{(f * \omega, \omega)\}]$, where $\omega(x)=e^{-x \cdot x}$.

THeORem 2.7. There exists a delta sequence $\left(\varphi_{n}\right)$ such that for every $T \in \mathscr{B}, T=\left[\left\{\left(f_{n}, \varphi_{n}\right)\right\}_{\mathbb{N}}\right]$ for some $f_{n} \in \mathcal{W}$.

Proof. Let $\left(\psi_{n}\right)$ be a delta sequence such that $\hat{\psi}_{n} \in \mathscr{D}$. Then, for any $T \in \mathscr{B}$, we have $\widehat{T} \hat{\psi}_{n} \in \mathscr{S}^{\prime}$, since $\hat{T} \in \mathscr{D}^{\prime}$. Consequently, $\widehat{T} \hat{\psi}_{n}=\widehat{g}_{n}$ for some $g_{n} \in \mathscr{S}^{\prime}$. It is easy to check that $T=\left[\left\{\left(g_{n} * \psi_{n}, \psi_{n} * \psi_{n}\right)\right\}_{\mathbb{N}}\right]$. Since $f_{n}=g_{n} * \psi_{n} \in \mathcal{W}$ and $\left(\varphi_{n}\right)=\left(\psi_{n} * \psi_{n}\right)$ is a delta sequence, the proof is complete. Note that $\left(\varphi_{n}\right)$ does not depend on $T$. 


\section{Algebraic properties and convergence}

$\mathscr{B}$ becomes a vector space with the operations defined as follows:

$$
\begin{gathered}
\lambda\left[\left\{\left(f_{j}, \varphi_{j}\right)\right\}_{J}\right]=\left[\left\{\left(\lambda f_{j}, \varphi_{j}\right)\right\}_{J}\right], \quad \lambda \in \mathbb{C}, \\
{\left[\left\{\left(f_{j}, \varphi_{j}\right)\right\}_{J}\right]+\left[\left\{\left(g_{k}, \psi_{k}\right)\right\}_{K}\right]=\left[\left\{\left(f_{j} * \psi_{k}+g_{k} * \varphi_{j}, \varphi_{j} * \psi_{k}\right)\right\}_{J \times K}\right] .}
\end{gathered}
$$

If $\left[\left\{\left(f_{j}, \varphi_{j}\right)\right\}_{j}\right],\left[\left\{\left(g_{k}, \psi_{k}\right)\right\}_{K}\right] \in \mathscr{B}$ and $g_{k} \in \mathscr{Y}$ for all $k \in K$, then we can define

$$
\left[\left\{\left(f_{j}, \varphi_{j}\right)\right\}_{J}\right] *\left[\left\{\left(g_{k}, \psi_{k}\right)\right\}_{K}\right]=\left[\left\{\left(f_{j} * g_{k}, \varphi_{j} * \psi_{k}\right)\right\}_{J \times K}\right] .
$$

It is easy to check that these operations are well defined. Note that, in view of Theorem 2.7, the definition of addition can be simplified.

Definition 3.1. Let $T_{0}, T_{1}, T_{2}, \ldots \in \mathscr{P}$. It is said that the sequence $\left(T_{n}\right)$ is convergent to $T_{0}$ and is written as $T_{n} \rightarrow T_{0}$ if there exists a total family $\left\{\varphi_{j}\right\}_{J}$ such that

(a) there exist tempered distributions $f_{j, n}$, where $j \in J$ and $n \in \mathbb{N}$, such that $T_{n}=$ $\left[\left\{f_{j, n}, \varphi_{j}\right\}_{J}\right]$ for all $n=0,1,2, \ldots$,

(b) $f_{j, n} \rightarrow f_{j, 0}$ in $\mathscr{S}^{\prime}$ as $n \rightarrow \infty$ for every $j \in J$.

Theorem 3.2. The Fourier transform is an isomorphism from $\mathscr{B}$ to $\mathscr{D}^{\prime}$.

Proof. Note that, since $T_{n} \rightarrow T_{0}$ in $\mathscr{B}$ if and only if $T_{n}-T_{0} \rightarrow 0$, it suffices to prove continuity at 0 .

Assume $T_{n} \rightarrow 0$ in $\mathscr{B}$. Then there exist tempered distributions $f_{j, n}$, where $j \in J$ and $n \in \mathbb{N}$, such that $T_{n}=\left[\left\{\left(f_{j, n}, \varphi_{j}\right)\right\}_{j}\right]$ for all $n=1,2, \ldots$ and $f_{j, n} \rightarrow 0$ in $\mathscr{Y}^{\prime}$ as $n \rightarrow \infty$ for every $j \in J$. If $\psi \in \mathscr{D}$, then there are $j_{1}, \ldots, j_{k}$ such that $\operatorname{supp} \psi \subset \bigcup_{m=1}^{k} \operatorname{supp} \hat{\varphi}_{j_{m}}$. Then

$$
\begin{aligned}
\lim _{n \rightarrow \infty} \widehat{T}_{n} \psi & =\lim _{n \rightarrow \infty} \sum_{m=1}^{k}\left(\hat{T}_{n} \hat{\varphi}_{j_{m}}\right) \frac{\overline{\hat{\varphi}_{j_{m}}} \psi}{\sum_{m=1}^{k}\left|\hat{\varphi}_{j_{m}}\right|^{2}} \\
& =\sum_{m=1}^{k}\left(\lim _{n \rightarrow \infty} \hat{f}_{j_{m}, n}\right) \frac{\overline{\hat{\varphi}_{j_{m}}} \psi}{\sum_{m=1}^{k}\left|\hat{\varphi}_{j_{m}}\right|^{2}}=0,
\end{aligned}
$$

since $\lim _{n \rightarrow \infty} \hat{f}_{j, n}=0$ for every $j \in J$, by continuity of the Fourier transform in $\mathscr{S}^{\prime}$. This proves continuity of $\mathscr{F}: \mathscr{B} \rightarrow \mathscr{D}^{\prime}$, because $\lim _{n \rightarrow \infty} \widehat{T}_{n} \psi=0$ in $\mathscr{Y}^{\prime}$ for every $\psi \in \mathscr{D}$ implies $\lim _{n \rightarrow \infty} \widehat{T}_{n}=0$ in $\mathscr{D}^{\prime}$.

Now assume $\lim _{n \rightarrow \infty} \widehat{T}_{n}=0$ in $\mathscr{D}^{\prime}$. By Theorem 2.7, there exists a delta sequence $\left(\varphi_{j}\right)$, $j \in \mathbb{N}$, such that for every $n \in \mathbb{N}$ we have $T_{n}=\left[\left\{\left(f_{j, n}, \varphi_{j}\right)\right\}_{\mathbb{N}}\right]$ for some $f_{j, n} \in \mathcal{W}$. Let $\left(\psi_{k}\right)$, $k \in \mathbb{N}$, be a delta sequence such that $\hat{\psi}_{k} \in \mathscr{D}$ for every $k \in \mathbb{N}$. Then $\lim _{n \rightarrow \infty} \widehat{T}_{n} \hat{\varphi}_{j} \hat{\psi}_{k}=0$ in $\mathscr{\varphi}^{\prime}$ for every $j, k \in \mathbb{N}$. Since $\widehat{T}_{n} \hat{\varphi}_{j}=f_{j, n}$ for every $j, k \in \mathbb{N}$, we have $\lim _{n \rightarrow \infty} \hat{f}_{j, n} \widehat{\psi}_{k}=0$ in $\mathscr{S}^{\prime}$, which implies $\lim _{n \rightarrow \infty} f_{j, n} * \psi_{k}=0$ in $\mathscr{Y}^{\prime}$. But

$$
T_{n}=\left[\left\{\left(f_{j, n}, \varphi_{j}\right)\right\}_{J}\right]=\left[\left\{\left(f_{j, n} * \psi_{k}, \varphi_{j} * \psi_{k}\right)\right\}_{J \times K}\right]
$$

for all $n=0,1,2, \ldots$, so we have $T_{n} \rightarrow 0$ in $\mathscr{B}$. 
2584 On the Fourier transform and the exchange property

\section{Acknowledgment}

The authors would like to thank Dennis Nemzer for helpful comments.

\section{References}

[1] D. Atanasiu, Fourier transform and the Boehme property, to appear in Integral Transform. Spec. Funct.

[2] V. Karunakaran and N. V. Kalpakam, Boehmians and Fourier transform, Integral Transform. Spec. Funct. 9 (2000), no. 3, 197-216.

[3] V. Karunakaran and R. Roopkumar, Ultra Boehmians and their Fourier transforms, Fract. Calc. Appl. Anal. 5 (2002), no. 2, 181-194.

[4] V. Karunakaran and V. B. Thilaga, Plancherel theorem for vector valued functions and Boehmians, Rocky Mountain J. Math. 28 (1998), no. 4, 1321-1342.

[5] V. Karunakaran and T. Venugopal, A new space for Fourier transform, Integral Transform. Spec. Funct. 9 (2000), no. 2, 133-148.

[6] P. Mikusiński, Fourier transform for integrable Boehmians, Rocky Mountain J. Math. 17 (1987), no. 3, 577-582.

[7] _ The Fourier transform of tempered Boehmians, Fourier Analysis (Orono, Me, 1992), Lecture Notes in Pure and Appl. Math., vol. 157, Dekker, New York, 1994, pp. 303-309.

[8] , Tempered Boehmians and ultradistributions, Proc. Amer. Math. Soc. 123 (1995), no. 3, 813-817.

[9] D. Nemzer, The Boehmians as an F-space, Ph.D. thesis, University of California, California, 1984.

[10] A. H. Zemanian, Distribution Theory and Transform Analysis, Dover, New York, 1987.

Dragu Atanasiu: School of Engineering, Borås University, Borås, SE 501 90, Sweden

E-mail address: dragu.atanasiu@hb.se

Piotr Mikusiński: Department of Mathematics, University of Central Florida, Orlando, FL 328161364, USA

E-mail address: piotrm@mail.ucf.edu 


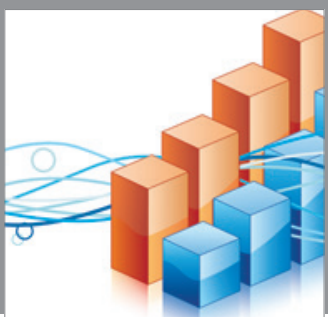

Advances in

Operations Research

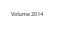

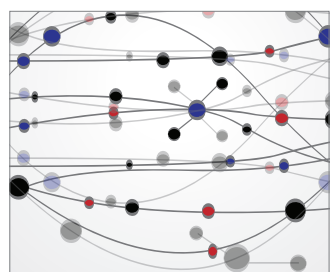

\section{The Scientific} World Journal
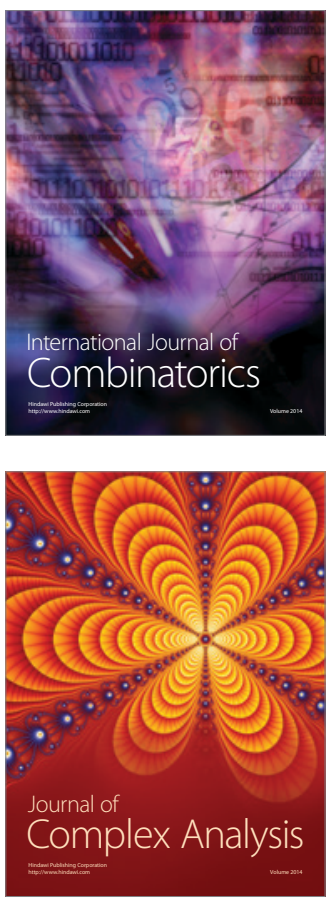

International Journal of

Mathematics and

Mathematical

Sciences
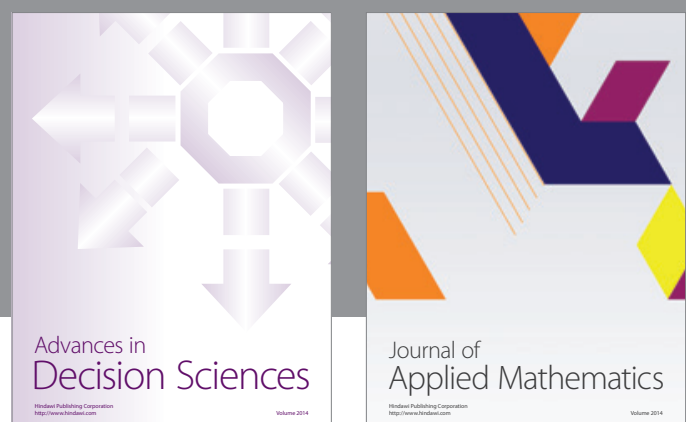

Journal of

Applied Mathematics
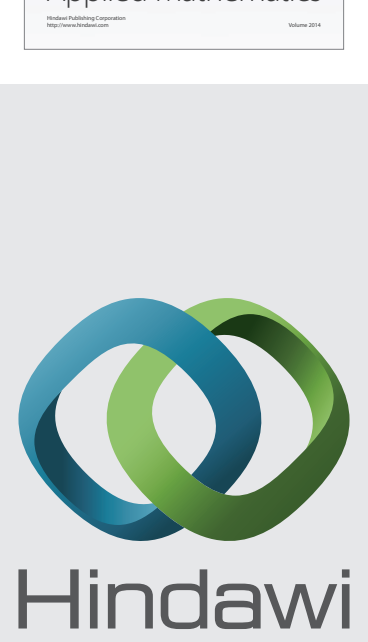

Submit your manuscripts at http://www.hindawi.com
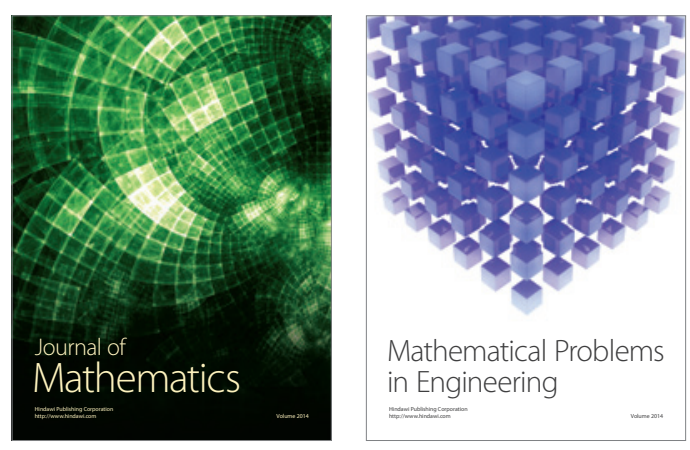

Mathematical Problems in Engineering
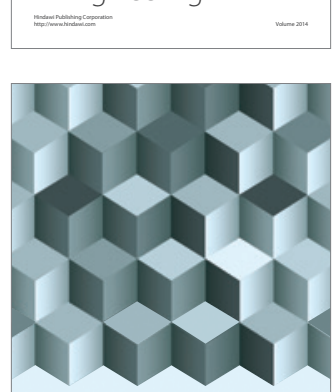

Journal of

Function Spaces
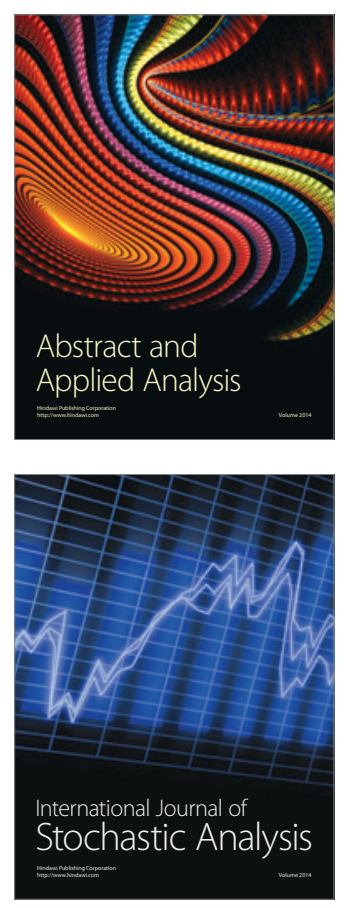

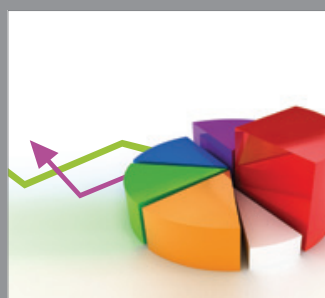

ournal of

Probability and Statistics

Promensencen
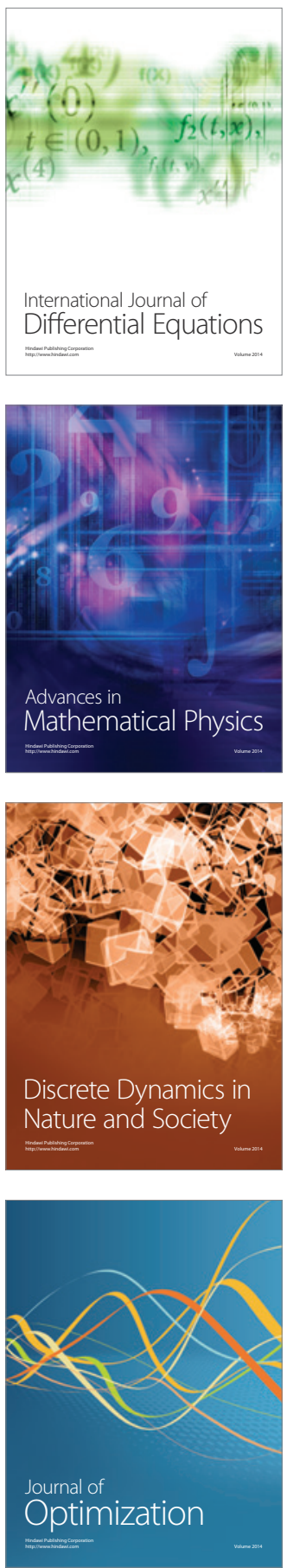